Monatsschrift f. Geburtshülfe u. Gynäkologie 1921;55:I-IV

\title{
Contents, Vol. 55, 1921
}

\section{Inhaltsverzeichnis.}

Seite

Baumm, Hans, Untersuchung uber die Gefährlichkeit

der manuellen Plazentarlösung 322

Dencker, Hans, Das Schicksal der Kaiserschnittkinder 207 Essen-Möller, Elis, Weitere

Eklampsieerfahrungen . 84 d'Erchia, F., Über Anatomie und Physiologie der Plazenta 65 Feis,

Oswald, Uber die Behandlung der ehronischen

weiblichen Gonorrhoe240

Frank, M., Über Pseudohermaphroditismus und zur Frage

des künstlichen Scheidenersatzes 5

Fraenkel, L. , Über Verletzungen des Spatium vesico

uterinum bei der Auí'stöpslung $\quad-340$

Füth, H., Beitrag zur Scheidenbildung nach Schubert. . 262 Geppert, F., Infarzierung des Uterus und der Adnexe . 346 Graebke, Heinr., Kasuistischer Beitrag zur Diagnose der

Nierentuberkulose in der Schwangerschaft und zur

Frage der Durchlässigkeit der Meren für Tuberkelbazillen 25 Hal ban, Josef, Zur Therapie des kombinierten Rektal-

und Genitalprolapses - 122

Haendly, P., Bestrahlung oder Operation? 239

Herfarth, H., Kongenitale Hypertrophie der Portio vagi-

nalis bei einem neugeborenen Kinde 125

Hinselmann, Hans, Subepitheliale Verdichtungsherde

im Bindegewebe der Blasenmolenzotten nebst einigen

allgemeineren Bemerkungen zur Blasenmolenforschung 1 Hannak, F., Über Prolapsus uteri et vaginae intra partum 233 Kirstein, F., Über die Deyke-Muchschen Partialantigene 218

Klaar, P., Ein neues Curettenmodell 349

Kofferath, W., Über einen Fall von rechtsseitiger Erbscher

Lähmung und Phrenikuslähmung nach Zangenextraktion 33 Krahula, Gerhard, Die Bedeutung des Hydramnion für

die Lebenserwartung des Kindes

Mayer, A., Die geburtshilfliche Bedeutung des durch zen-

trale Schenkelluxation verengten Beckens 315

Offermann, Walter, Neues Material zur Behandlung

septischer Aborte 99

Oertel, Christian, Über zwei atypische Geburtsvorgänge

bei einem engen Becken 266

P rib ram, E., Zur Tuberkulose der Ovarialgeschwülste . 256 Rating, J o h., Zwei Fälle von puerperalem Mammaerysipel 129 Riediger, K., Neue Erfahrungen mit der Kiellandschen Zange 113 
Salomon, R., Endogener Mikrobismus

331

Schroeder, E., Schwere atypische Entbindung. - Missed'

labour. - Symphysenruptur intra partum 103

- IV -

Seite

S c h r ö d e r , R., Über die Pathogenese der Uterustuberkulose 15 Unterberger, F.,

Experimentelle Untersuchungen über

ektopische Dezidua 116

Aus den Grenzgebieíen.

Heinsius. F.. Soil der Staat ärztliche Heiratszeugnisse

fordern? ' 274

Schmidt man n, M., Eklampsie und chronische Nephritis 132

Sammelbericht über die französische Literatur 1918-1919.

Von Dr. U. Zanolari in Zurich

145

Medizinische Eindrücke meiner dritten südamerikanischen

Reise. Von A. Dührssen 153

Vereins- und Literaturbeiiage. Oríginal-Siízungsberichte aus geburtshülf lich-gynäkologischen

Gesellschaften.

Nordostdeutsche Gesellschaft für Gynäkologie. Sitzung vom

26. II. $1921 \ldots$, $\quad 157$

Dresdner Gvnäkologische Gesellschaft. Sitzung vom 21. X..

18. XI., 16. XII. 1920 und 21. I. 1921; 17. II., 17. III.

und 21. IV. $1921 \quad$ 171. 295

Niederrheinisch-westfälische Gesellschaft für Gynäkologie

und Geburtshülfe. 64. Sitzung vom 7. V. 1921 . . . 279 Gemeinsame Tagung der Ober-, Mittel-

und Niederrheinischen

Gesellschaft für Geburtshülfe und Gynäkologie am 25.

und 26. IV. in Bad Kreuznach 294

Gynäkologische Gesellschaft in Breslau. Sitzuiigen vom

25. I., 22. II., 8. III. 1921 und 31. V. 1921 . . . 40, 354 Gesellschaft für Geburtshülfe und

Gynäkologie zu Berlin.

Sitzung vom 11. III. und 21. IV. 1921 “ 177

45. Versammlung der Deutschen Gesellschaft für Chirurgie

vom 30. III. bis 2. IV. 1921179

Referate aus geburtshülflich-gynäkologischen Gesellschaften

55, 183, 298, 364

Geburtshülflich-gynäkologische Mitteîlungen aus anderen medi-

zínischen Gesellschaften und Vereinen . . 56, 186, 299, 364

Buchbesprechungen 57, 187, 300, 365

Literaturverzeichnïs 62, 192, 307, 368

Wilhelm Tauffer (2. Juli 1851-1921) 38

Ernst Fraenkel † 19. März 156

Personalien 39, 314 